\section{Pesquisa em psicanálise e psicoterapia psicanalítica: um novo instrumento para avaliação de aderência à técnica em estudos de efetividade}

Research in psychoanalysis and psychoanalytic psychotherapy: a new tool for the evaluation of adherence to the technique in studies of effectiveness

\footnotetext{
Sr. Editor,

O surgimento de diferentes opções terapêuticas e o embasamento das condutas em evidências científicas têm levado à melhora do atendimento psiquiátrico, possibilitando o tratamento eficaz de uma série de psicopatologias. No entanto, um número significativo de pacientes permanece com sintomas residuais, necessitando de abordagens que vão além da melhora sintomática. Tradicionalmente utilizada para o alívio do sofrimento psíquico e ampliação da capacidade adaptativa, a técnica psicanalítica pode ter papel fundamental, quando corretamente indicada. Apesar de corroborada por mais de 100 anos de experiência clínica e
}

pesquisas que vão desde relatos de caso a estudos de seguimento, a utilidade da técnica psicanalítica dentro da medicina moderna tem sido questionada, frente à possibilidade de tratamentos que seriam eficazes em menor espaço de tempo e de menor custo. De fato, apesar do número crescente de pesquisas na área psicanalítica, diversos autores apontam que não há consenso, do ponto de vista da medicina baseada em evidências, sobre como os tratamentos psicanalíticos funcionam, para quem funcionam e em quais condições. ${ }^{1-3}$

A eficácia da utilização de aplicações do modelo psicanalítico em tratamentos breves manualizados de uma série de patologias específicas já foi sistematicamente comprovada, ${ }^{4,5}$ mas a problemática do uso da manualização e do modelo clássico de eficácia na investigação da efetividade dos tratamentos psicanalíticos "tradicionais" tem sido questionada. Conforme discutido por Maat et al., "modelos híbridos", que utilizem desenho naturalístico, aliados a parâmetros que visem garantir a validade interna, têm sido sugeridos. ${ }^{6}$ Nesse sentido, são necessárias ferramentas que se proponham a medir aderência à técnica utilizada sem a necessidade de manualização. Com esse objetivo, foi desenvolvido o Instrumento para Avaliação de Sessões Psicanalíticas (IASP).

A etapa inicial na elaboração do IASP consistiu-se em ampla revisão da literatura e discussões em grupos focais, visando apreender aspectos que definiriam uma sessão como psicanalítica. Após esta etapa, foram realizados sucessivos testes de aplicação que englobaram sessões de psicoterapeutas psicanalíticos com diversos níveis de formação e "sessões modelo" de terapia interpessoal (TIP) e cognitivo-comportamental (TCC). Foram realizadas uma série de análises fatoriais e testes de confiabilidade ao longo dessa etapa. A versão final resultante contém cinco itens, que englobam: neutralidade do terapeuta, natureza das intervenções, realização de interpretações, uso da teoria para compreensão do material, criação de espaços reflexivos e aspectos da relação paciente-terapeuta.

Ao longo do processo, foi elaborada e testada uma folha de rosto com instruções de preenchimento. Testes preliminares da versão final demonstraram boa confiabilidade (Alpha Cronbah's $=0,81$ ), apresentando apenas um fator, com todos os itens tendo participação relevante na sua composição (Análise dos Componentes Principais). A confiabilidade entre os avaliadores foi boa (Kappa $=0,81$ ). Nessa etapa, foram avaliadas 36 sessões de psicoterapia psicanalítica, bem como sessões modelo de TIP e TCC, tendo participado oito avaliadores, sendo que dois deles não tinham conhecimento prévio do instrumento.

O IASP está sendo utilizado em estudos em andamento na Universidade Federal do Rio Grande do Sul e sua performance está sendo avaliada em circunstâncias diversas. Está disponível para utilização em pesquisas mediante solicitação (shauck@hcpa.org.br).

Simone Hauck Serviço de Psiquiatria, Hospital de Clínicas de Porto Alegre, Porto Alegre (RS), Brasil Departamento de Psiquiatria e Medicina Legal, Universidade Federal do Rio Grande do Sul (UFRGS), Porto Alegre (RS), Brasil

Programa de Pós-Graduação em Ciências Médicas, Universidade Federal do Rio Grande do Sul (UFRGS), Porto Alegre (RS), Brasil

Tiago Crestana, Clarissa Kristen Mombach, Éllen Alves de Almeida Hospital de Clínicas de Porto Alegre, Porto Alegre (RS), Brasil 
Financiamento e conflito de interesses

\begin{tabular}{|c|c|c|c|c|c|c|c|}
\hline $\begin{array}{l}\text { Membro do grupo } \\
\text { de autores }\end{array}$ & $\begin{array}{l}\text { Local de } \\
\text { trabalho }\end{array}$ & $\begin{array}{l}\text { Verba de } \\
\text { pesquisa }\end{array}$ & $\begin{array}{l}\text { Outro apoio à pesquisa ou } \\
\text { educação médica continuada }\end{array}$ & $\begin{array}{l}\text { Honorários de } \\
\text { palestrante }\end{array}$ & $\begin{array}{c}\text { Participação } \\
\text { acionária }\end{array}$ & $\begin{array}{c}\text { Consultor/ Conselho } \\
\text { consultivo }\end{array}$ & Outro $^{3}$ \\
\hline Simone Hauck & HCPA & - & - & - & - & - & - \\
\hline Tiago Crestana & HCPA & - & - & - & - & - & - \\
\hline $\begin{array}{l}\text { Clarissa Kristen } \\
\text { Mombach }\end{array}$ & HCPA & - & - & - & - & - & - \\
\hline $\begin{array}{l}\text { Ellen Alves de } \\
\text { Almeida }\end{array}$ & HCPA & - & - & - & - & - & - \\
\hline Cláudio Laks Eizirik & $\begin{array}{l}\text { HCPA } \\
\text { UFRGS }\end{array}$ & $\begin{array}{l}\text { FIPE- } \\
\text { HCPA }\end{array}$ & - & - & - & - & - \\
\hline
\end{tabular}

* Modesto

** Significativa

*** Significativa. Montantes fornecidos à instituição do autor ou a colega para pesquisa onde o autor tem participação, não diretamente ao autor.

Nota: HCPA = Hospital de Clinicas de Porto Alegre; UFRGS = Universidade Federal do Rio Grande do Sul; FIPE = Fundo de Incentivo à Pesquisa e Ensino.

Para mais informações, consultar as Instruçōes aos autores.

\section{Cláudio Laks Eizirik}

Serviço de Psiquiatria, Hospital de Clínicas de Porto Alegre,

Porto Alegre (RS), Brasil

Departamento de Psiquiatria e Medicina Legal, Universidade Federal do Rio Grande do Sul (UFRGS),

Porto Alegre (RS), Brasil

Programa de Pós-Graduação em Ciências Médicas, Universidade Federal do Rio Grande do Sul (UFRGS),

Porto Alegre (RS), Brasil

References

1. Gabbard GO, Gunderson JG, Fonagy P. The place of psychoanalytic treatments within psychiatry. Arch Gen Psychiatry. 2002;59(6):505-10.

2. Eizirik CL, Hauck S. Psicanálise e Psicoterapia Psicanalítica. In: Cordioli AV, editor. Psicoterapias: abordagens atuais. 3a ed. Porto Alegre: Artmed; 2008. p. 155-62.

3. Eizirik CL. Psychoanalysis and research. Rev Bras Psiquiatr. 2006;28(3):171-2.

4. Leichsenring F, Rabung S, Leibing E. The efficacy of short-term psychodynamic psychotherapy in specific psychiatric disorders. Arch Gen Psychiatry. 2004;61(12):1208-16.

5. Leichsenring F, Leibing E. Psychodynamic psychotherapy: a systematic review of techniques, indications and empirical evidence. Psychol Psychotherapy. 2007;80(Pt 2):217-28.

6. Maat SD, Dekker J, Schoevers R, Jonghe FD. The effectiveness of long-term psychotherapy: methodological research issues. Psychother Res. 2007;17(1):59-65. 